\title{
Management of adhesive capsulitis
}

This article was published in the following Dove Press journal:

Orthopedic Research and Reviews

24 August 2015

Number of times this article has been viewed

\section{Kristen L Stupay'}

Andrew S Neviaser ${ }^{2}$

'Tulane University School of Medicine, New Orleans, LA, USA; ${ }^{2}$ George Washington University Medical Faculty Associates, Washington, DC, USA
Correspondence: Andrew S Neviaser George Washington University Medical Faculty Associates, 2150 Pennsylvania Ave, NW \#5, Washington, DC 20037, USA Email aneviaser@gmail.com
Abstract: Adhesive capsulitis of the shoulder is a condition of capsular contracture that reduces both active and passive glenohumeral motion. The cause of adhesive capsulitis is not known but it is strongly associated with endocrine abnormalities such as diabetes. Diverse terminology and the absence of definitive criteria for diagnosis make evaluating treatment modalities difficult. Many treatment methods have been reported, most with some success, but few have been proved to alter the natural course of this disease. Most afflicted patients will achieve acceptable shoulder function without surgery. Those who remain debilitated after 8-12 months are reasonable candidates for invasive treatments. Here, the various treatment methods and the data to support their use are reviewed.

Keywords: frozen shoulder, stiff shoulder, periarthritis, painful shoulder

\section{Introduction Epidemiology}

Adhesive capsulitis is a disabling condition characterized by the spontaneous onset of motion loss about the shoulder. It affects up to $5 \%$ of the population, ${ }^{1,2}$ occurs primarily in people between the ages of 40 and 60 years, and has been seen to disproportionately affect females. ${ }^{3-5}$ Patients affected by endocrine disorders, such as diabetes, ${ }^{6-8}$ thyroid disease, ${ }^{9-11}$ and autoimmune disease, ${ }^{1,12,13}$ also seem to be affected more often than those of the general population. Hypercholesterolemia and inflammatory lipoproteinemias have also been shown to be significantly associated with the development of primary adhesive capsulitis. ${ }^{14}$

\section{Natural history}

Most clinical accounts indicate that this condition is self-limiting and will typically resolve in 2-3 years. A retrospective review by Vastamäki et al evaluated 51 patients diagnosed with adhesive capsulitis who received no treatment, and found that after a mean duration of 15 months of symptoms (range: 4-36 months), 94\% of patients regained their normal range of motion. ${ }^{15}$ However, movement restrictions have been shown to persist far beyond this time point; a 2008 study on the long-term outcomes of adhesive capsulitis reported that, at a mean follow-up of 4.4 years, $41 \%$ of 269 cases were still symptomatic. ${ }^{15}$ In this same study, the authors observed a $92 \%$ and $89 \%$ return-to-normal rate in patients treated nonoperatively or with manipulation, respectively, indicating that more severe cases requiring treatment may not fare as well in the long term. ${ }^{15}$ 


\section{Terminology}

The term "frozen shoulder" was coined initially by Codman to describe a common constellation of symptoms that included a "slow onset [...] of pain felt near the insertion of the deltoid, inability to sleep on the affected side, painful and restricted elevation and external rotation, with a normal radiological appearance" ${ }^{16}$ In 1945 , Neviaser identified synovial inflammation and a thickened, contracted capsule that seemed to adhere to the humeral head, as well as to opposed synovium as the main cause of motion loss. The pathology described correlated clinically with early pain and muscle spasm (synovitis) followed by profound stiffness (adhesions and contracture). It was also at this time that the frozen shoulder was given its more descriptive and appropriate title, "adhesive capsulitis". ${ }^{17}$

\section{Pathology}

The etiology of adhesive capsulitis is not known, but is understood to involve a sequence of synovial inflammation, hyperplasia, and subsequent capsular fibrosis. ${ }^{18,19}$ Four predictable and overlapping stages of this disease occur. ${ }^{17,20-22}$ This allows for disease staging upon diagnosis, which is essential to successful patient counseling and symptom management.

Synovitis and capsular contracture as defining features have been confirmed using both arthrographic and arthroscopic techniques. ${ }^{23,24}$ The pathognomonic findings on arthroscopy include obliteration of the subscapularis recess with scar tissue covered by a highly vascular synovium, as well as a tightened axillary recess with reduced joint volume. ${ }^{1}$ Thickening and contracture of the coracohumeral ligament is another consistently reported feature of adhesive capsulitis..$^{25,26}$

Neviaser and Neviaser defined the arthroscopic appearance of the shoulder joint throughout this disease process, and established the four stages that we now use to clinically stage the disease. ${ }^{21}$ In 1994, Hannafin et al demonstrated the correlating histopathological characteristics in synovial specimens taken from patients in the various Neviaser stages. ${ }^{22}$ Neither arthroscopic exam nor histopathology is routinely used to diagnose or stage the disease, but their description provides us with a greater understanding of the pathophysiology of this condition. A detailed description of the arthroscopic and histological features of this disease are described elsewhere..$^{20,21}$

\section{Biochemistry}

Although the arthroscopic and histological evidence points to an inflammatory and subsequent fibrotic cascade, the particular biochemical processes unfolding in the shoulder joint throughout these four stages are not fully understood. The predominant theory is that stimulation of an inflammatory synovitis eventually results in a fibrotic cascade, culminating in the thickened and contracted capsule that characterizes the later stages of the disease. ${ }^{27}$ Several studies aim to identify the cytokines, cell types, and growth factors involved in this cycle. In 1997, Rodeo et al performed immunohistochemical localization on capsule and synovial biopsies taken from patients with adhesive capsulitis, patients with nonspecific synovitis, and patients with normal capsule. ${ }^{19}$ This evaluation revealed new type III collagen deposition in the adhesive capsulitis patients, as well as fibroblastic proliferation and the presence of platelet derived growth factor, interleukin-1 beta, and tumor necrosis factor-alpha in both those with adhesive capsulitis and in nonspecific synovitis. The patients who had normal capsules did not stain for these substances. These findings were supported by Bunker, who examined the excised capsular tissue of 12 patients who underwent open capsular release for treatment of manipulation-refractory primary adhesive capsulitis. Bunker, too, found that the capsular tissue was made of thick, mature type III collagen. ${ }^{1}$ These studies all support the notion that inflammation, followed by a subsequent fibrotic cascade, is responsible for the clinical entity of adhesive capsulitis.

\section{Diagnosis}

When conceptualizing adhesive capsulitis, it is helpful to delineate into two types based on the presence or absence of concomitant shoulder disease. Primary or idiopathic adhesive capsulitis refers to the disease process in isolation, when the progressive pain and stiffness occur without an identifiable cause. Secondary adhesive capsulitis refers to capsular contracture that is indistinguishable from idiopathic adhesive capsulitis pathologically, but which occurs secondary to a soft-tissue injury, fracture, arthritis, or hemiplegia; postsurgically; or due to another known lesion. While many circumstances both intrinsic and extrinsic to the shoulder joint can lead to pain and stiffness, adhesive capsulitis is a distinct pathological process and should not be conflated with loss of shoulder range of motion and pain associated with postoperative stiffness.

Distinguishing between primary and secondary adhesive capsulitis is useful only in that it helps us to organize the various pathways by which patients can be affected by this condition; however, it provides little true assistance in disease diagnosis or management. ${ }^{28}$ The diagnosis of idiopathic adhesive capsulitis in clinical practice is one based on history and physical exam. 
Early diagnosis is preferable as there are interventions that may be undertaken to reduce morbidity; however, this can be rather challenging as the pain of early-stage adhesive capsulitis is difficult to distinguish from that of impingement or rotator cuff disease. ${ }^{29}$ In a recent study on treatment of primary idiopathic frozen shoulder, only $17 \%$ of 850 referrals to the investigators met the criteria for true adhesive capsulitis, highlighting the challenge in diagnosing this condition. ${ }^{27}$

There is currently no gold standard for diagnosis, nor have validated diagnostic criteria been published in the literature. ${ }^{30,31}$ In 2009, Walmsley et al used a validated technique to query a panel of 70 experts on the clinical signs and symptoms indicative of the first stage of primary adhesive capsulitis. ${ }^{29}$ The panel came to consensus on eight clinical identifiers to aid in diagnosing adhesive capsulitis in its early stages. They include: a strong component of night pain; a marked increase in pain with rapid or unguarded movements; discomfort as a result of lying on the affected shoulder; pain that is easily aggravated by movement; onset in people $>35$ years old; global loss of passive glenohumeral joint movement, and global loss of active and passive range of movement, as well as pain at the end range in all directions on exam. Despite expert consensus on these criteria, a subsequent study by the same group was unable to validate the utility of these identifiers in diagnosing stage 1 disease. ${ }^{30}$

\section{History}

When assessing for adhesive capsulitis, the history should elicit the onset and duration of symptoms, presence of night or rest pain, "jerk" pain with sudden movements, and functional limitation, as well as associated medical conditions. As the disease progresses from pain to painful stiffness, patients typically report difficulty with dressing, combing their hair, and internally rotating to fasten a bra or access their back pocket. Later, when stiffness is predominant, range of motion will be significantly limited in all directions, and pain may only occur when the shoulder is moved beyond the limits of the contracted capsule. ${ }^{32}$

\section{Physical exam}

The physical exam should include a full evaluation of the cervical spine, trunk, and shoulders. Patients in stage 1 or 2 may have pain on palpation of the anterior and posterior capsule, with pain that radiates toward the insertion of the deltoid. ${ }^{27,33}$ Performing a full evaluation of active and passive range of motion is necessary to help determine the presenting stage of the disease, and should be documented so as to assess efficacy of treatment.

\section{Special tests}

There have been no validated specialized tests to identify adhesive capsulitis, though two recent studies have described physical exam maneuvers that may help the examiner to detect the disease early on. ${ }^{33,34}$ It has been repeatedly observed that external rotation - particularly in abduction - is the most limited movement in adhesive capsulitis. ${ }^{31}$ In 2010, Wolf and Cox sought to exploit this observation, and described the "external rotation test". They found that of 91 patients presenting with a positive external rotation test, $75 \%$ were later clinically diagnosed with adhesive capsulitis. The only other diagnosis that produced a positive external rotation test was glenohumeral arthritis, and these patients all had commensurate crepitus on exam and radiological evidence of arthritis on plain X-ray. ${ }^{34}$

In 2014, Noboa et al described a new diagnostic test, which they called the Distension Test in Passive External Rotation (DTPER). ${ }^{33}$ In their series of patients, the DTPER showed high sensitivity (100\% with $95 \%$ confidence interval) and specificity ( $90 \%$ with $95 \%$ confidence interval) for adhesive capsulitis. These tests have yet to be validated by any other studies. ${ }^{35}$

\section{Imaging}

Routine radiographs should be obtained to rule out other potential causes of a stiff and painful shoulder. Occasionally, there may be osteopenia of the humeral head and neck, ${ }^{35}$ but plain X-rays in adhesive capsulitis should be otherwise normal. The use of ultrasound, magnetic resonance imaging, or magnetic resonance arthrography is rarely necessary, but the finding using these modalities has been described. Ultrasound offers a low-cost, dynamic assessment imaging modality that may reveal increased vascular flow and thickening of the rotator interval in stage 1 disease. An edematous and thick axillary pouch and a thickened coracohumeral ligament are classic findings on magnetic resonance imaging. Utilizing T2-weighted images and contrast enhancement highlights specific soft-tissue areas in the shoulder that are sensitive and specific for identification of adhesive capsulitis. ${ }^{36-39}$

\section{Treatment}

The majority of treatment options for adhesive capsulitis are nonoperative and include pharmacological management and physical therapy. Many other treatment methods have been tried with limited success. A multimodal, nonoperative treatment program is effective for most patients. ${ }^{39}$ For those who do not improve with nonoperative measures, opera- 
tive management includes manipulation under anesthesia (MUA) or arthroscopic capsular release.

\section{Nonoperative treatment}

\section{Nonsteroidal anti-inflammatory drugs}

Although use of nonsteroidal anti-inflammatory drugs (NSAIDs) for analgesia in almost any stage of the disease is a widely accepted intervention, it should be noted that there are no studies in the literature validating the use of NSAIDs over placebo or other oral agents. For earlystage adhesive capsulitis, when pain is the predominant symptom, oral nonsteroidal anti-inflammatory medications may be initiated. A double-blinded, parallel study by Rhind et al compared the use of naproxen and indomethacin two times per day for 4 weeks in 41 patients with adhesive capsulitis. Both analgesics reduced pain to a significant degree; however, neither drug improved objective range-of-motion deficit, and there was an appreciable incidence of side effects (nausea, headache) experienced by the participants. As a result, three patients in the naproxen group and five in the indomethacin group stopped treatment. ${ }^{40}$ In another study that evaluated the same drugs at higher doses, similar results were seen and four of the 59 patients withdrew from this study secondary to side effects. ${ }^{41}$

\section{Oral steroids}

Oral steroids may be given to relieve pain and stiffness. There are several older studies that provide high-level evidence on oral steroids as compared with placebo, no treatment, or other treatments. In a 1954 double-blind, randomized controlled trial of 30 patients, Blockey et al compared placebo with a cortisone acetate suspension taper over a period of 4 weeks. All patients were instructed to perform rigorous home exercise as well. Although pain and range of motion were believed to have improved earlier in the steroid group, no significant differences in pain or range of motion were seen at 18 weeks' follow-up. ${ }^{42}$ A second double-blind, randomized controlled trial of 49 patients compared $30 \mathrm{mg}$ per day doses of prednisolone with placebo pills over a period of 3 weeks. All patients in this study were given pendulum and scapular retraction exercises to perform at home. At 6 and 12 weeks' follow-up, there was no significant difference in pain, range of motion, or shoulder-specific disability. Similar to the Blockey et al study, earlier improvements in pain and function were seen in the prednisolone group; however, after cessation of treatment, the patients in the steroid group either failed to improve further or worsened, whereas the placebo group showed consistent improvement throughout the 12 -week period. ${ }^{43}$

In another study comparing oral steroids with no treatment, patients in the experimental group were given $10 \mathrm{mg}$ prednisolone for 4 weeks, followed by $5 \mathrm{mg}$ for 2 weeks. All patients were instructed to perform daily pendulum exercises at home. At 5-month follow-up, pain was significantly decreased in the treatment group relative to the control group; however, this benefit was not sustained at 8 months. ${ }^{44}$

Several low-level studies performed more recently have supported these early findings, and collectively suggest that oral steroids do have the potential to provide short-term benefits in pain, range of shoulder motion, and shoulder function, but that these benefits are not maintained over the course of the disease process. ${ }^{45,46}$ The use of oral steroids to quickly relieve symptoms must be weighed against the risks of systemic steroid use. ${ }^{43}$

\section{Intra-articular injections}

Intra-articular injections of corticosteroid and local anesthetics may have some short-term utility in treating pain and improving motion in adhesive capsulitis. There is extensive literature available on intra-articular corticosteroids, and recently hyaluronic acid and botulinum toxin injections have also emerged as potential alternatives.

\section{Corticosteroids}

Intra-articular injections of corticosteroid have been extensively studied and are known to provide pain relief and motion improvement early in the pathological process of adhesive capsulitis. When given in stage 1 , when pain is most severe, the anti-inflammatory properties of corticosteroid effectively reduce inflammation, thereby reducing pain, spasm, and stiffness. If given during stage 2, the pain relief is often still significant, though capsular contracture at this stage prevents improved movement. ${ }^{47}$ There is a large body of evidence that supports the use of corticosteroid in the relief of pain in early-stage adhesive capsulitis; however, several recent systematic reviews of high-level evidence emphasize that, while corticosteroids are effective in providing short-term relief, they typically have no lasting effect relative to other treatment modalities. ${ }^{48,49}$

Bulgen et al assessed the differences in outcomes at 6 months between groups that received either an intraarticular methylprednisolone injection, mobilization with a physical therapist, ice after proprioceptive exercises, or no treatment. All patients were taught pendulum exercises to perform at home. Duration of symptoms at presentation 
ranged from 1 to 12 months (mean, 4.8 months); however, they did not report stage at the time of intervention. At 4-week follow-up, there was no difference in pain symptoms between the groups; however, the corticosteroid group had the most significant range-of-motion improvement. At final follow-up, the difference between groups was no longer significant and all patients reported improved pain at 6 months. ${ }^{50}$

In 1991, Rizk et al compared injection of methylprednisolone plus lidocaine with that of a lidocaine placebo injection, delivered either into the glenohumeral joint or the subacromial bursa. All patients underwent physical therapy. In order to be included, participants could not have had symptoms for more than 6 months. Weekly follow-up was carried out for the first 11 weeks, and then again at 15 weeks and 6 months. There was no significant difference seen in pain or range of motion; however, the corticosteroid groups did experience pain relief more quickly than did the placebo groups. ${ }^{51}$

van der Windt et al randomized 109 patients into two groups to receive either $40 \mathrm{mg}$ of intra-articular triamcinolone acetonide or physiotherapy twice per week for 6 weeks. They did not report the duration of symptoms or stage at time of diagnosis. At 7 weeks' follow-up, $77 \%$ of patients in the corticosteroid group were considered a success, compared with $46 \%$ of the physiotherapy group. This finding was statistically significant, and remained so at 52 weeks' follow-up. ${ }^{52}$

In 2003, Carette et al conducted a randomized, placebocontrolled trial comparing four groups: 1) fluoroscopically guided intra-articular triamcinolone hexacetonide plus physiotherapy; 2) triamcinolone hexacetonide injection alone; 3) saline injection plus physiotherapy; and 4) saline injection alone. All patients also performed a simple home exercise program. There were 93 patients included in this study, all of whom had less than 1 year's duration of symptoms at the time of intervention. At 6 weeks and 3 months, shoulder disability had improved significantly more in the steroid-injection groups than in the groups that did not receive steroids. Range-of-motion measurements increased in all groups, with the steroid-plus-physiotherapy group improving significantly more than all the other groups at 6 weeks. By 1 year, however, there was no statistically significant difference in any of the outcomes between any of the groups. $^{53}$

In a level II randomized controlled trial, Roh et al compared outcomes of triamcinolone intra-articular injection plus a home stretching routine to that of a control group who performed home stretching alone. Forty-five patients were included in this study, all of which were diabetics. Although range of motion and pain were significantly improved in the injection group over the non-injection group in the very short term (3-6 weeks), there was no significant difference in either outcome between the groups at 6 months. ${ }^{54}$

In 2013, Yoon et al published a triple-blind randomized placebo-controlled trial of 53 patients comparing intraarticular injections of high-dose triamcinolone acetonide plus lidocaine, low-dose triamcinolone plus lidocaine, and lidocaine alone. All patients in this study were clinically identified as being in stage 2 , and after a single injection were expected to carry out a home exercise program. All three groups showed significant improvement of outcome measures with time alone. Improvement in shoulder-specific disability, pain, and range of motion improved significantly more in both corticosteroid groups than in the placebo group; however, there were no statistically significant differences in outcomes between the high-dose and low-dose groups. The authors concluded that low-dose corticosteroid injection should be encouraged as initial treatment so as to minimize potential local and systemic complications. ${ }^{55}$

Despite the lack of long-term improvement to outcomes, the anti-inflammatory and pain-reducing properties of corticosteroid make injection of these substances a valuable addition to the treatment regimen of patients with earlystage adhesive capsulitis, allowing for improved activity, sleep, and overall quality of life in the more painful stages of this disease.

\section{Hyaluronic acid}

In vitro studies have shown hyaluronic acid has the propensity to modulate cell proliferation and expression of mRNA adhesion-related procollagens and cytokines, suggesting that it may prevent progression of adhesion formation in adhesive capsulitis. ${ }^{56}$ In two high-level studies, hyaluronic acid has been evaluated as a potential alternative to corticosteroids. In 1998, Rovetta and Monteforte demonstrated that patients receiving a combination injection of hyaluronate and triamcinolone fared better functionally than those who had received corticosteroid alone. ${ }^{57}$

Later, Calis et al compared pain, function, and range-ofmotion improvement following randomization to one of four groups: 1) injection with sodium hyaluronate; 2) injection with triamcinolone acetonide; 3 ) a combination of physical therapy modalities for 10 days; or 4) no treatment. All groups were advised to carry out home stretching and Codman exercises. The hyaluronic acid group performed similarly to those given corticosteroid injection in all categories except for external rotation values at 2 weeks. It is known that hyaluronic acid does not begin to take effect until approximately 2 weeks 
following injection, however, and by 3 months, this rotational difference was no longer appreciable. ${ }^{58}$

These results were repeated in a recent randomized, prospective study of 68 patients who received either hyaluronic acid or corticosteroid injection; no significant differences were seen between the groups save for internal rotation at 2 weeks, a difference that resolved by 3 months. ${ }^{59}$ Similarly, a 2011 systematic review including four level I studies and three level IV studies concluded that clinical outcomes of hyaluronic acid injections are superior to controls and equivalent to intraarticular corticosteroid injections in the short term. ${ }^{60}$

Interestingly, two randomized trials have found that injection with hyaluronic acid did not show significant benefit over patients receiving physical therapy alone. ${ }^{58,61}$ Hyaluronic acid injections, therefore, continue to produce mixed results.

\section{Botulinum toxin}

Botulinum toxin type A (BoNT-A) has been seen in a histological in vivo study to prevent joint fibrosis and adhesions, as well as to have inhibitory effects on pain-mediating neuropeptides and central sensitization. ${ }^{62,63}$ It has recently been considered as another possible alternative to injected corticosteroids for adhesive capsulitis.

In a prospective, controlled trial of 28 patients in the "painful freezing phase", both corticosteroid and BoNT-A groups saw improvement in shoulder range of motion and pain relief. Though Joo et al found no significant difference between the two groups in short-term pain relief, they did find a significant improvement in both active and passive abduction of the shoulder in those who received BoNT-A. The authors postulate that this difference may be attributable to more effective pain relief by BoNT-A in the early stages of adhesive capsulitis. ${ }^{62}$

In summary, several studies indicate that intra-articular injections of corticosteroid that are delivered during the first or second stage of disease provide symptom relief in the short term that is not sustained at longer-term follow-up. ${ }^{56,57,63-65}$ Both hyaluronic acid and BoNT-A injections may be safe, albeit more expensive, alternatives to corticosteroids that could find use in patients who are unable to tolerate the side effects of a corticosteroid, or as a second-line option in those whose disease does not respond to corticosteroids alone.

\section{Physical therapy}

Physical therapy is commonly prescribed for adhesive capsulitis regardless of the stage of disease; however, the literature has failed to produce conclusive results on these interventions. Blanchard et al performed a systematic review of six randomized controlled trials and concluded that, in the short term, corticosteroid injections have a greater effect than physical therapy on improving pain and disability, but this difference was not sustained over time. ${ }^{66}$ To the contrary, a more recent systematic review by Jain and Sharma established a strong recommendation for the use of therapeutic exercises and mobilization in patients with stage 2 and 3 adhesive capsulitis to reduce pain, improve range of motion, and increase function. ${ }^{67}$ Despite mixed results, most providers do not question the value of a well-designed physical therapy program in treatment, and multiple studies have documented treatment success in reducing pain or increasing mobility. ${ }^{58,68}$

Griggs et al prospectively followed 75 patients with stage 2 adhesive capsulitis who underwent a four-direction shoulder-stretching exercise program. Stretching in this program was not designed to push the patients beyond minimal discomfort. These patients were followed up for a mean duration of 22 months, and $90 \%$ of the patients reported a satisfactory outcome. ${ }^{69}$

In a prospective study of 77 patients, Diercks and Stevens compared intensive physical therapy consisting of passive stretching and manual mobilization with "supervised neglect", which included pendulum and active exercises in the pain-free range. All the patients had had symptoms for greater than 3 months. At 24 months' follow-up, 89\% of the patients in the supervised neglect group had normal or nearnormal painless shoulder function, as compared with only $63 \%$ of the patients in the intensive physical therapy group. Although supervised neglect is a somewhat misleading term to describe the interventions, this study suggests that painfree exercises are better than exercises that stretch beyond the pain threshold, and that pushing strenuous exercise may actually worsen the long-term prognosis. ${ }^{70}$

This finding was not supported in a randomized controlled trial comparing the effects of high-grade versus low-grade mobilization techniques on shoulder-specific disability and range of motion. One hundred patients with a mean of 8 months of symptoms underwent 12 weeks of physical therapy. High-grade mobilization included intensive passive stretching at end-range of motion, whereas low-grade techniques focused on passive motion within the pain-free zone. The authors concluded that, although high-grade mobilization was superior in improving joint mobility and functional ability, at 1 -year follow-up there was very minimal overall difference between these two treatment strategies. ${ }^{71}$ 
In a blinded randomized controlled trial by Russell et al, 75 patients underwent either 1) group exercise therapy plus home exercises; 2) individualized physiotherapy plus home exercises; or 3) home exercises alone for 6 weeks. The mean duration of symptoms at presentation was 5.79 months. All three groups showed significant improvement in pain and function at 6 weeks, 6 months, and 1 year, but the group exercise therapy produced a faster functional recovery than in either of the other two groups. At 1 year, group exercise therapy showed significant improvement in Constant and Oxford shoulder scores over both individual physiotherapy and home exercises. The authors postulated that the shorter recovery times and better patient-reported outcomes in the group class may be attributable to peer support and motivation. ${ }^{27}$

However successful physical therapy has been in curbing short-term morbidity, there has been no high-level evidence to suggest that it has any effect on overall disease prognosis. ${ }^{72}$ Still, the existing literature suggests that stretching and active motion that does not cause significant pain to the patient is adequate to improve pain, range of motion, and function in the short term.

\section{Suprascapular nerve blocks}

The suprascapular nerve arises from the brachial plexus and provides motor innervation to the supraspinatus and infraspinatus muscles and sensory innervation to the glenohumeral and acromioclavicular joints. A practice initially conducted by anesthesiologists, suprascapular nerve blocks have shown some positive results in a small number of studies for use in treating adhesive capsulitis. The mechanism by which this works is unclear, but, theoretically, disrupting the pain feedback loop in the shoulder joint allows for recalibration of pathological and neurological processes that ultimately reduces pain. Reduced pain, in turn, allows for improved function. $^{73}$

In 1999, Jones and Chattopadhyay conducted a randomized trial of 30 patients to assess the efficacy of a single suprascapular nerve block with triamcinolone and bupivacaine as compared to a series of up to three intra-articular injections of triamcinolone and lidocaine. All patients recruited to participate were in stage 2 or 3 of adhesive capsulitis and all were advised on a home exercise program to participate in following the injections. Both treatment groups showed marked improvement in pain and range of motion, but the suprascapular nerve block group demonstrated more rapid pain relief and a significantly greater reduction in pain and improvement in range of motion at 3 months. ${ }^{73}$
In 2000, Dahan et al conducted a double-blind randomized placebo-controlled trial comparing two groups of 17 patients after receiving suprascapular nerve blocks with bupivacaine versus injections with normal saline placebo. All 34 subjects underwent three injections each at 7-day intervals. The mean duration of pain prior to randomization was 1 year. At 2 weeks after the last injection, $62 \%$ of the patients in the bupivacaine group experienced a significant improvement in pain as compared with $13 \%$ in the placebo group. Shoulder function was improved in both groups, and there was no improvement in range of motion seen in either group. ${ }^{74}$

Although these studies are somewhat promising, larger comparative studies with more thorough follow-up are needed. This technique could be particularly useful in allowing for proper rehabilitation in the early postoperative period.

\section{Hydrodilation}

Hydrodilation, or capsular distention, is the injection of large amounts of fluid into the joint with the intent of increasing capsular volume and pressure, subsequently causing capsule rupture. This is a fairly simple procedure that does not require general anesthesia and can be performed by either anterior or posterolateral approach. ${ }^{75}$ As such, it is a viable alternative to operative intervention for adhesive capsulitis. ${ }^{76-78}$

Ahn et al compared ultrasound-guided capsular distension with ketorolac to corticosteroid injection in 121 patients and found that both modalities provided equivalent pain relief and functional improvement in the short term; however, the capsular distension group showed superior improvements in range of motion at 3 and 6 months' follow-up. ${ }^{79}$ Quraishi et al conducted a randomized controlled trial of 38 shoulders comparing hydrodilation with MUA. Those who underwent MUA also received an intra-articular injection of triamcinolone. The mean duration of symptoms at the time of treatment was 33.7 weeks, and the patients were followed up at 2 and 6 months after hydrodilation. The Constant scores and pain scores improved significantly more in the hydrodilation group than in the MUA group. No differences were noted in range of motion. ${ }^{80}$

A recent prospective randomized controlled trial evaluated the effectiveness of hydrodilation in combination with either intensive mobilization therapy, corticosteroid injection, or both in patients with stage 1 or 2 adhesive capsulitis. Perhaps unsurprisingly, after 4 weeks of treatment, the group that combined all three modalities experienced greater improvement in pain, active range of motion, and shoul- 
der function than the groups receiving fewer therapeutic approaches. The authors hypothesized that the combination addressed inflammation with the corticosteroid, extended the joint space with capsular distension, and recovered range of motion with intensive mobilization. ${ }^{81}$

To date, there is no consensus regarding the necessity of capsular rupture for the success of this procedure. Recently, studies have looked at whether preservation of capsular integrity leads to better outcomes. Kim et al used pressurevolume curves to monitor for capsular rupture during distension procedures in 46 patients, and found that the patients in whom the capsule was preserved saw enhanced short-term outcomes, though long-term outcomes between the groups were not different. ${ }^{82}$

There are relatively few studies published on the outcomes and effectiveness of hydrodilation. ${ }^{83}$ A Cochrane Review from 2008 was unable to draw definitive conclusions about the utility of the procedure, but there is some evidence that it provides short-term and long-term benefits in pain, range of motion, and function in adhesive capsulitis. ${ }^{84}$

\section{Operative treatment Closed MUA}

Closed MUA is a technique that has proved effective in treating primary adhesive capsulitis at both short- and long-term follow-up, regardless of symptom duration. ${ }^{85}$ Recent evidence has indicated that the optimal timing for manipulation may be between 6 and 9 months following symptom onset. ${ }^{86}$ Despite good short- and long-term outcomes, it is important to note that the complication profile of MUA risks significant damage to the glenohumeral joint and surrounding structures. ${ }^{87-91}$ Contraindications to this procedure include significant osteopenia, recent surgical repair of soft tissues about the shoulder, or presence of fracture or neurologic injury.

Dodenhoff et al prospectively assessed 39 shoulders treated with MUA. The mean time from symptom onset to MUA was 8 months, and all of the patients included had stage 2 disease with lessening pain. Mean Constant scores as well as range of motion both showed significant improvement at 6 months and 12 months following manipulation. At final follow-up, $94 \%$ of patients reported satisfaction with the procedure. ${ }^{92}$ Despite apparently high patient satisfaction, a randomized and controlled trial of 125 patients in 2007 concluded that, although there were slight, insignificant advantages in range-of-motion gain in the manipulation group, no additional pain or functional benefit was gained by adding MUA to a home physical therapy program. ${ }^{93}$
Farrell et al evaluated the long-term results of MUA in 26 patients for whom nonoperative management failed to restore motion. These patients had undergone physical therapy for a mean of 6.2 months prior to undergoing MUA. The authors were able to collect long-term data on 19 of the 26 shoulders at a mean of 15 years from the time of intervention. Eighteen of the 19 shoulders never required surgery, and 16 shoulders were reported to cause slight or no pain at final follow-up. ${ }^{90}$ Vastamäki and Vastamäki reported similarly positive results at a mean of 23 years' follow-up. ${ }^{91}$

For patients who experience range-of-motion restriction that is refractory to physical therapy and other nonoperative treatment modalities, MUA can be a safe and effective procedure that provides lasting pain relief. Complications are a legitimate concern with this procedure, but can be minimized if contraindications are respected and if it is performed using the proper technique. ${ }^{94,95}$

\section{Arthroscopic capsular release}

Arthroscopy has in recent years become the preferred method of treatment for adhesive capsulitis that has not responded to nonoperative treatment. It not only allows for confirmation of the diagnosis and release of the capsule without the risks inherent to manipulation, but also provides an opportunity for the surgeon to evaluate and potentially treat concomitant glenohumeral or subacromial disease.

Arthroscopic intervention can be adjunctive to MUA, either to confirm diagnosis and document manipulation success, or to perform post-manipulation debridement of the capsule. Some authors advocate for these combined methods over closed MUA or arthroscopic release alone. ${ }^{96,97}$

De Carli et al randomized 46 patients in stage 2 adhesive capsulitis refractory to NSAIDs and physical therapy to receive either manipulation followed by arthroscopic capsular release or intra-articular injection of methylprednisolone. All the patients in both groups showed significant and satisfactory results at 1 year postintervention; however, the combined manipulation-arthroscopy group started gaining significant range of motion 6 weeks earlier than those in the intra-articularinjection group..$^{97}$ Pollock et al reported similar results in their study of 30 shoulders that underwent arthroscopic debridement of the rotator interval following MUA. Eighty-three percent of the shoulders yielded satisfactory results in this series. ${ }^{98}$

Arthroscopy may also be used to facilitate the precise release of all or part of the capsule in lieu of manipulation. Releasing the capsule arthroscopically has been shown in multiple studies to improve shoulder range of motion and function and decrease the time to full recovery. ${ }^{99-103}$ Ogilvie- 
Harris et al conducted a prospective cohort study on 40 patients who underwent either manipulation preceded and followed by arthroscopic exam or arthroscopic capsular release alone. Both groups performed similarly on range of motion at long-term follow-up; however, the arthroscopic division group had significantly better pain relief and significantly fewer functional deficits as compared to the manipulation group. ${ }^{104}$

In a 2014 prospective study on arthroscopic release, Mehta et al compared outcomes between patients who had diabetes mellitus and those who did not. All patients recruited were in stage 2 , and had undergone a trial of nonoperative management prior to arthroscopic release. Both groups improved significantly with regards to Constant scores, range of motion, and pain at 2-year follow-up. Nondiabetic patients had significantly better Constant scores at 6 weeks and 6 months postoperatively than diabetic patients, a difference that was not appreciated at 2 years. This suggests that diabetic patients undergoing arthroscopic release take a longer time to restore function, but in the long-term perform similarly to nondiabetic patients. ${ }^{105}$ This finding is consistent with previous literature on the treatment of diabetic patients. ${ }^{104}$

There has also been the question as to which aspects of the capsule should be released. Some advocate for circumferential release, whereas others endorse a more selective process that only releases the posterior capsule if anterior and inferior release fails to produce satisfactory results. ${ }^{106,107}$ Chen et al conducted a level I therapeutic randomized controlled trial of 74 patients undergoing arthroscopic capsular release which found no significant difference at 6 months in Constant scores, pain, or range of motion between patients who had release of the anterior capsule only versus those who underwent anterior, inferior, and posterior release. ${ }^{108}$ Despite lack of consensus in this aspect, this procedure consistently shows sustained improvement in pain, function, and range of motion at long-term follow-up. ${ }^{109,110}$

A 2013 article by Dattani et al sought to evaluate the functional and health-related quality-of-life outcomes following circumferential arthroscopic capsular release. One hundred patients were included in this study, all of whom had failed nonoperative treatment and were identified to be in the "stiffness-predominant" phase. All patients participated in weekly physical therapy for 4 months following release. Range of motion and shoulder function improved significantly by 6 months. The authors were able to calculate the mean quality-adjusted life year for arthroscopic capsular release, which allowed them to assess the relative value of this procedure from an economic perspective. They concluded that arthroscopic capsular release was a very cost-effective intervention for the treatment of adhesive capsulitis; however, they were careful to note that the cost of MUA is in fact lower. ${ }^{111}$

There are few high-level trials comparing MUA to arthroscopic capsular release for operative treatment of adhesive capsulitis, and the data available demonstrate little benefit for arthroscopic capsular release over or in addition to MUA. ${ }^{112}$ Despite this, arthroscopic release is currently the preferred method for treatment as patients seem to have equivalent if not better pain and range-of-motion improvements in short- and long-term periods with a lower risk of complications. ${ }^{27,29,110}$

\section{Open capsular release}

Open capsular release for the treatment of adhesive capsulitis is a procedure that is rarely performed. The indications are very few, and are limited to patients who have been unsuccessful with closed manipulation and arthroscopic capsular release. Omari and Bunker reported excellent or good results in 20 of 25 of patients who underwent open capsular release. All of these patients did not improve with nonoperative management and failed release with manipulation. ${ }^{13}$ Ozaki et al achieved excellent results in 17 patients undergoing surgical release of the contracted coracohumeral ligament. ${ }^{26}$ Both groups highlighted the importance of reserving this technique for patients whose adhesive capsulitis was severe and refractory to all other treatment, and urged caution in patients with insulin-dependent diabetes mellitus.

\section{Conclusion}

Adhesive capsulitis can be a prolonged and disabling condition characterized by inflammation and fibrosis of the shoulder joint capsule leading to pain and restricted shoulder motion. Early diagnosis of this condition is challenging due to its nonspecific presentation. Paying close attention to the duration and onset of symptoms as well as limitation of motion, particularly external rotation and abduction, may help to differentiate early adhesive capsulitis from other painful and stiff conditions of the shoulder. Managing adhesive capsulitis requires that the provider identify the approximate stage of disease at presentation, and use this information to guide treatment. Generally, patients presenting in the early, pain-predominant stages should undergo gentle physical therapy that focuses on motion that is not overly painful. Physical therapy may be complemented with oral NSAIDs and intra-articular injections of corticosteroid, both of which have been shown to provide 
relief of pain early and therefore should be used whenever possible to reduce morbidity in the short term, but have not been shown to improve symptom duration or prognosis. Patients who do not improve with nonoperative measures and those who present in the stiffness-predominant stages may be candidates for more invasive procedures such as capsular distension, MUA, or arthroscopic capsular release. Arthroscopic capsular release is considered the preferred method, as it allows for confirmation of diagnosis and precise capsular release, as well as treatment of any coexisting shoulder conditions.

\section{Disclosure}

The authors report no conflicts of interest in this work.

\section{References}

1. Bunker TD. Frozen shoulder: unravelling the enigma. Ann R Coll Surg Engl. 1997;79(3):210-213.

2. van der Windt DA, Koes BW, de Jong BA, Bouter LM. Shoulder disorders in general practice: incidence, patient characteristics, and management. Ann Rheum Dis. 1995;54(12):959-964.

3. Tekavec E, Jöud A, Rittner R, et al. Population-based consultation patterns in patients with shoulder pain diagnoses. BMC Musculoskelet Disord. 2012;13:238.

4. Hand C, Clipsham K, Rees JL, Carr AJ. Long-term outcome of frozen shoulder. J Shoulder Elbow Surg. 2008;17(2):231-236.

5. Walker-Bone K, Palmer KT, Reading I, Coggon D, Cooper C. Prevalence and impact of musculoskeletal disorders of the upper limb in the general population. Arthritis Rheum. 2004;51(4):642-651.

6. Bridgman JF. Periarthritis of the shoulder and diabetes mellitus. Ann Rheum Dis. 1972;31(1):69-71.

7. Sayed-Hassan R, Alourfi Z. Shoulder adhesive capsulitis prevalence among patients with type 2 diabetes mellitus in Damascus, Syrian Arab Republic: a case-control study. East Mediterr Health J. 2014; 19 Suppl 3:S19-S24.

8. Wang K, Ho V, Hunter-Smith DJ, Beh PS, Smith KM, Weber AB. Risk factors in idiopathic adhesive capsulitis: a case control study. J Shoulder Elbow Surg. 2013;22(7):e24-e29.

9. Huang SW, Lin JW, Wang WT, Wu CW, Liou TH, Lin HW. Hyperthyroidism is a risk factor for developing adhesive capsulitis of the shoulder: a nationwide longitudinal population-based study. Sci Rep. 2014;4:4183.

10. Bowman CA, Jeffcoate WJ, Pattrick M, Doherty M. Bilateral adhesive capsulitis, oligoarthritis and proximal myopathy as presentation of hypothyroidism. Br J Rheumatol. 1988;27(1):62-64.

11. Wohlgethan JR. Frozen shoulder in hyperthyroidism. Arthritis Rheum. 1987;30(8):936-939.

12. Bulgen DY, Binder A, Hazleman BL, Park JR. Immunological studies in frozen shoulder. J Rheumatol. 1982;9(6):893-898.

13. Smith SP, Devaraj VS, Bunker TD. The association between frozen shoulder and Dupuytren's disease. J Shoulder Elbow Surg. 2001;10(2): 149-151.

14. Sung CM, Jung TS, Park HB. Are serum lipids involved in primary frozen shoulder? A case-control study. J Bone Joint Surg Am. 2014; 96(21):1828-1833.

15. Vastamäki H, Kettunen J, Vastamäki M. The natural history of idiopathic frozen shoulder: a 2- to 27-year followup study. Clin Orthop Relat Res. 2012;470(4):1133-1143.

16. Codman EA, ed. Tendinitis of the short rotators. In: Ruptures of the Supraspinatus Tendon and Other Lesions in or About the Subacromial Bursa. Boston: Thomas Todd and Co; 1934.
17. Neviaser JS. Adhesive capsulits of the shoulder: a study of pathological findings in periarthritis of the shoulder. J Bone Joint Surg Am. 1945;27: 211-212.

18. Harris G, Bou-Haidar P, Harris C. Adhesive capsulitis: review of imaging and treatment. J Med Imaging Radiat Oncol. 2013;57(6):633-643.

19. Rodeo SA, Hannafin JA, Tom J, Warren RF, Wickiewicz TL. Immunolocalization of cytokines and their receptors in adhesive capsulitis of the shoulder. J Orthop Res. 1997;15(3):427-436.

20. Neviaser JS. Arthrography of the shoulder joint: study of the findings in adhesive capsulitis of the shoulder. Study of the findings in adhesive capsulitis of the shoulder. J Bone Joint Surg Am. 1962;44-A: 1321-1359.

21. Neviaser RJ, Neviaser TJ. The frozen shoulder. Diagnosis and management. Clin Orthop Relat Res. 1987;(223):59-64.

22. Hannafin JA, DiCarlo EF, Wickiewicz TL. Adhesive capsulits: capsular fibro-plasia of the glenohumeral joint. J Shoulder Elbow Surg. 1994;3(1):S5.

23. Wiley AM. Arthroscopic appearance of frozen shoulder. Arthroscopy. 1991;7(2):138-143.

24. Uitvlugt G, Detrisac DA, Johnson LL, Austin MD, Johnson C. Arthroscopic observations before and after manipulation of frozen shoulder. Arthroscopy. 1993;9(2):181-185.

25. Neer CS 2nd, Satterlee CC, Dalsey RM, Flatow EL. The anatomy and potential effects of contracture of the coracohumeral ligament. Clin Orthop Relat Res. 1992;(280):182-185.

26. Ozaki J, Nakagawa Y, Sakurai G, Tamai S. Recalcitrant chronic adhesive capsulitis of the shoulder. Role of contracture of the coracohumeral ligament and rotator interval in pathogenesis and treatment. J Bone Joint Surg Am. 1989;71(10):1511-1515.

27. Russell S, Jariwala A, Conlon R, Selfe J, Richards J, Walton M. A blinded, randomized, controlled trial assessing conservative management strategies for frozen shoulder. J Shoulder Elbow Surg. 2014;23(4): 500-507.

28. Kelley MJ, Shaffer MA, Kuhn JE, et al. Shoulder pain and mobility deficits: adhesive capsulitis. J Orthop Sports Phys Ther. 2013;43(5):A1-A31.

29. Walmsley S, Rivett DA, Osmotherly PG. Adhesive capsulitis: establishing consensus on clinical identifiers for stage 1 using the DELPHI technique. Phys Ther. 2009;89(9):906-917.

30. Walmsley S, Osmotherly PG, Rivett DA. Clinical identifiers for earlystage primary/idiopathic adhesive capsulitis: are we seeing the real picture? Phys Ther. 2014;94(7):968-976.

31. Walmsley S, Osmotherly PG, Rivett DA. Movement and pain patterns in early stage primary/idiopathic adhesive capsulitis: a factor analysis. Physiotherapy. 2014;100(4):336-343.

32. Neviaser AS, Neviaser RJ. Adhesive capsulitis of the shoulder. J Am Acad Orthop Surg. 2011;19:536-542.

33. Noboa E, López-Graña G, Barco R, Antuña S. Distension test in passive external rotation: validation of a new clinical test for the early diagnosis of shoulder adhesive capsulitis. Rev Esp Cir Ortop Traumatol. Epub December 17, 2014. English, Spanish.

34. Wolf EM, Cox WK. The external rotation test in the diagnosis of adhesive capsulitis. Orthopedics. 2010;33(5).

35. Binder AI, Bulgen DY, Hazleman BL, Tudor J, Wraight P. Frozen shoulder: an arthrographic and radionuclear scan assessment. Ann Rheum Dis. 1984;43(3):365-369.

36. Gokalp G, Algin O, Yildirim N, Yazici Z. Adhesive capsulitis: contrastenhanced shoulder MRI findings. J Med Imaging Radiat Oncol. 2011; 55(2):119-125.

37. Carbone S, Napoli A, Gumina S. MRI of adhesive capsulitis of the shoulder: distension of the bursa in the superior subscapularis recess is a suggestive sign of the pathology. Eur J Radiol. 2014;83(2): 345-348.

38. Gondim Teixeira PA, Balaj C, Chanson A, Lecocq S, Louis M, Blum A. Adhesive capsulitis of the shoulder: value of inferior glenohumeral ligament signal changes on T2-weighted fat-saturated images. AJR Am J Roentgenol. 2012;198(6):W589-W596.

39. Rill BK, Fleckenstein CM, Levy MS, Nagesh V, Hasan SS. Predictors of outcome after nonoperative and operative treatment of adhesive capsulitis. Am J Sports Med. 2011;39(3):567-574. 
40. Rhind V, Downie WW, Bird HA, Wright V, Engler C. Naproxen and indomethacin in periarthritis of the shoulder. Rheumatol Rehabil. 1982;21(1):51-53.

41. Duke O, Zecler E, Grahame R. Anti-inflammatory drugs in periarthritis of the shoulder: a double-blind, between-patient study of naproxen versus indomethacin. Rheumatol Rehabil. 1981;20(1):54-59.

42. Blockey NJ, Wright JK, Kellgren JH. Oral cortisone therapy in periarthritis of the shoulder; a controlled trial. Br Med J. 1954;1(4877): 1455-1457.

43. Buchbinder R, Green S, Youd JM, Johnston RV. Oral steroids for adhesive capsulitis. Cochrane Database Syst Rev. 2006;(4):CD006189.

44. Binder A, Hazleman BL, Parr G, Roberts S. A controlled study of oral prednisolone in frozen shoulder. Br J Rheumatol. 1986;25(3):288-292.

45. Canbulat N, Eren I, Atalar AC, Demirhan M, Eren SM, Ucak A. Nonoperative treatment of frozen shoulder: oral glucocorticoids. Int Orthop. 2015;39(2):249-254.

46. Takase K. Oral steroid therapy for frozen shoulder. West Indian Med J. 2010;59(6):674-679.

47. Neviaser AS, Hannafin JA. Adhesive capsulitis: a review of current treatment. Am J Sports Med. 2010;38(11):2346-2356.

48. Buchbinder R, Green S, Youd JM. Corticosteroid injections for shoulder pain. Cochrane Database Syst Rev. 2003;(1):CD004016.

49. Griesser MJ, Harris JD, Campbell JE, Jones GL. Adhesive capsulitis of the shoulder: a systematic review of the effectiveness of intra-articular corticosteroid injections. J Bone Joint Surg Am 2011;93(18):1727-1733.

50. Bulgen DY, Binder AI, Hazleman BL, Dutton J, Roberts S. Frozen shoulder: prospective clinical study with an evaluation of three treatment regimens. Ann Rheum Dis. 1984;43(3):353-360.

51. Rizk TE, Pinals RS, Talaiver AS. Corticosteroid injections in adhesive capsulitis: investigation of their value and site. Arch Phys Med Rehabil. 1991;72(1):20-22.

52. van der Windt DA, Koes BW, Devillé W, Boeke AJ, de Jong BA, Bouter LM. Effectiveness of corticosteroid injections versus physiotherapy for treatment of painful stiff shoulder in primary care: randomised trial. BMJ. 1998;317(7168):1292-1296.

53. Carette S, Moffet H, Tardif J, et al. Intraarticular corticosteroids, supervised physiotherapy, or a combination of the two in the treatment of adhesive capsulitis of the shoulder: a placebo-controlled trial. Arthritis Rheum. 2003;48(3):829-838.

54. Roh YH, Yi SR, Noh JH, et al. Intra-articular corticosteroid injection in diabetic patients with adhesive capsulitis: a randomized controlled trial. Knee Surg Sports Traumatol Arthrosc. 2012;20(10):1947-1952.

55. Yoon SH, Lee HY, Lee HJ, Kwack KS. Optimal dose of intra-articular corticosteroids for adhesive capsulitis: a randomized, triple-blind, placebo-controlled trial. Am J Sports Med. 2013;41(5):1133-1139.

56. Nago M, Mitsui Y, Gotoh M, et al. Hyaluronan modulates cell proliferation and mRNA expression of adhesion-related procollagens and cytokines in glenohumeral synovial/capsular fibroblasts in adhesive capsulitis. J Orthop Res. 2010;28(6):726-731.

57. Rovetta G, Monteforte P. Intraarticular injection of sodium hyaluronate plus steroid versus steroid in adhesive capsulitis of the shoulder. Int $J$ Tissue React. 1998;20(4):125-130.

58. Calis M, Demir H, Ulker S, Kirnap M, Duygulu F, Calis HT. Is intraarticular sodium hyaluronate injection an alternative treatment in patients with adhesive capsulitis? Rheumatol Int. 2006;26(6):536-540.

59. Lim TK, Koh KH, Shon MS, Lee SW, Park YE, Yoo JC. Intra-articular injection of hyaluronate versus corticosteroid in adhesive capsulitis. Orthopedics. 2014;37(10):e860-e865.

60. Harris JD, Griesser MJ, Copelan A, Jones GL. Treatment of adhesive capsulitis with intra-articular hyaluronate: a systematic review. Int $J$ Shoulder Surg. 2011;5(2):31-37.

61. Hsieh LF, Hsu WC, Lin YJ, Chang HL, Chen CC, Huang V. Addition of intra-articular hyaluronate injection to physical therapy program produces no extra benefits in patients with adhesive capsulitis of the shoulder: a randomized controlled trial. Arch Phys Med Rehabil. 2012;93(6): 957-964.
62. Joo YJ, Yoon SJ, Kim CW, et al. A comparison of the short-term effects of a botulinum toxin type a and triamcinolone acetate injection on adhesive capsulitis of the shoulder. Ann Rehabil Med. 2013;37(2): 208-214.

63. Song A, Higgins LD, Newman J, Jain NB. Glenohumeral corticosteroid injections in adhesive capsulitis: a systematic search and review. PMR. 2014;6(12):1143-1156.

64. Ryans I, Montgomery A, Galway R, Kernohan WG, McKane R. A randomized controlled trial of intra-articular triamcinolone and/or physiotherapy in shoulder capsulitis. Rheumatology (Oxford). 2005; 44(4):529-535.

65. Gross C, Dhawan A, Harwood D, Gochanour E, Romeo A. Glenohumeral joint injections: a review. Sports Health. 2013;5(2):153-159.

66. Blanchard V, Barr S, Cerisola FL. The effectiveness of corticosteroid injections compared with physiotherapeutic interventions for adhesive capsulitis: a systematic review. Physiotherapy. 2010;96(2):95-107.

67. Jain TK, Sharma NK. The effectiveness of physiotherapeutic interventions in treatment of frozen shoulder/adhesive capsulitis: a systematic review. J Back Musculoskelet Rehabil. 2014;27(3):247-273.

68. Arslan S, Celiker R. Comparison of the efficacy of local corticosteroid injection and physical therapy for the treatment of adhesive capsulitis. Rheumatol Int. 2001;21(1):20-23.

69. Griggs SM, Ahn A, Green A. Idiopathic adhesive capsulitis. A prospective functional outcome study of nonoperative treatment. J Bone Joint Surg Am. 2000;82-A(10):1398-1407.

70. Diercks RL, Stevens M. Gentle thawing of the frozen shoulder: a prospective study of supervised neglect versus intensive physical therapy in seventy-seven patients with frozen shoulder syndrome followed up for two years. J Shoulder Elbow Surg. 2004;13(5):499-502.

71. Vermeulen HM, Rozing PM, Obermann WR, le Cessie S, Vliet Vlieland TP. Comparison of high-grade and low-grade mobilization techniques in the management of adhesive capsulitis of the shoulder: randomized controlled trial. Phys Ther. 2006;86(3):355-368.

72. Struyf F, Meeus M. Current evidence on physical therapy in patients with adhesive capsulitis: what are we missing? Clin Rheumatol. 2014; 33(5):593-600.

73. Jones DS, Chattopadhyay C. Suprascapular nerve block for the treatment of frozen shoulder in primary care: a randomized trial. $\mathrm{Br} J \mathrm{Gen}$ Pract. 1999;49(438):39-41.

74. Dahan TH, Fortin L, Pelletier M, Petit M, Vadeboncoeur R, Suissa S. Double blind randomized clinical trial examining the efficacy of bupivacaine suprascapular nerve blocks in frozen shoulder. J Rheumatol. 2000;27(6):1464-1469.

75. Bae JH, Park YS, Chang HJ, et al. Randomized controlled trial for efficacy of capsular distension for adhesive capsulitis: fluoroscopy-guided anterior versus ultrasonography-guided posterolateral approach. Ann Rehabil Med. 2014;38(3):360-368.

76. Rizk TE, Gavant ML, Pinals RS. Treatment of adhesive capsulitis (frozen shoulder) with arthrographic capsular distension and rupture. Arch Phys Med Rehabil. 1994;75(7):803-807.

77. Bell S, Coghlan J, Richardson M. Hydrodilatation in the management of shoulder capsulitis. Australas Radiol. 2003;47(3):247-251.

78. Vad VB, Sakalkale D, Warren RF. The role of capsular distention in adhesive capsulitis. Arch Phys Med Rehabil. 2003;84(9):1290-1292.

79. Ahn JK, Kim J, Lee SJ, Park Y, Cho S, Lee WY. Effects of ultrasound guided capsular distension with ketorolac in frozen shoulder. $J$ Back Musculoskelet Rehabil. Epub October 15, 2014.

80. Quraishi NA, Johnston P, Bayer J, Crowe M, Chakrabarti AJ. Thawing the frozen shoulder. A randomised trial comparing manipulation under anaesthesia with hydrodilatation. J Bone Joint Surg Br. 2007;89(9): 1197-1200.

81. Park SW, Lee HS, Kim JH. The effectiveness of intensive mobilization techniques combined with capsular distension for adhesive capsulitis of the shoulder. J Phys Ther Sci. 2014;26(11):1767-1770.

82. Kim K, Lee KJ, Kim HC, Lee KJ, Kim DK, Chung SG. Capsule preservation improves short-term outcome of hydraulic distension in painful stiff shoulder. J Orthop Res. 2011;29(11):1688-1694. 
83. Clement RG, Ray AG, Davidson C, Robinson CM, Perks FJ. Frozen shoulder: long-term outcome following arthrographic distension. Acta Orthop Belg. 2013;79(4):368-374.

84. Buchbinder R, Green S, Youd JM, Johnston RV, Cumpston M. Arthrographic distension for adhesive capsulitis (frozen shoulder). Cochrane Database Syst Rev. 2008;(1):CD007005.

85. Thomas WJ, Jenkins EF, Owen JM, et al. Treatment of frozen shoulder by manipulation under anaesthetic and injection: does the timing of treatment affect the outcome? J Bone Joint Surg Br. 2011;93(10): 1377-1381.

86. Vastamäki H, Varjonen L, Vastamäki M. Optimal time for manipulation of frozen shoulder may be between 6 and 9 months. Scand J Surg. Epub January 26, 2015.

87. Ahmad D, Hashim JA, Asim HM. Outcome of manipulation under anaesthesia in adhesive capsulitis patients. J Coll Physicians Surg Pak. 2014;24(4):293-294.

88. Wang JP, Huang TF, Ma HL, Hung SC, Chen TH, Liu CL. Manipulation under anaesthesia for frozen shoulder in patients with and without non-insulin dependent diabetes mellitus. Int Orthop. 2010;34(8):1227-1232.

89. Loew M, Heichel TO, Lehner B. Intraarticular lesions in primary frozen shoulder after manipulation under general anesthesia. J Shoulder Elbow Surg. 2005;14(1):16-21.

90. Farrell CM, Sperling JW, Cofield RH. Manipulation for frozen shoulder: long-term results. J Shoulder Elbow Surg. 2005;14(5):480-484.

91. Vastamäki H, Vastamäki M. Motion and pain relief remain 23 years after manipulation under anesthesia for frozen shoulder. Clin Orthop Relat Res. 2013;471(4):1245-1250.

92. Dodenhoff RM, Levy O, Wilson A, Copeland SA. Manipulation under anesthesia for primary frozen shoulder: effect on early recovery and return to activity. J Shoulder Elbow Surg. 2000;9(1):23-26.

93. Kivimäki J, Pohjolainen T, Malmivaara A, et al. Manipulation under anesthesia with home exercises versus home exercises alone in the treatment of frozen shoulder: a randomized, controlled trial with 125 patients. J Shoulder Elbow Surg. 2007;16(6):722-726.

94. Sharma S. Management of frozen shoulder - conservative vs surgical? Ann R Coll Surg Engl. 2011;93(5):343-344; discussion 345-346.

95. Atoun E, Funk L, Copland SA, Even T, Levy O, Rath E. The effect of shoulder manipulation on rotator cuff integrity. Acta Orthop Belg. 2013;79(3):255-259.

96. Andersen NH, Søjbjerg JO, Johannsen HV, Sneppen O. Frozen shoulder: arthroscopy and manipulation under general anesthesia and early passive motion. J Shoulder Elbow Surg. 1998;7(3):218-222.

97. De Carli A, Vadalà A, Perugia D, et al. Shoulder adhesive capsulitis: manipulation and arthroscopic arthrolysis or intra-articular steroid injections? Int Orthop. 2012;36(1):101-106.
98. Pollock RG, Duralde XA, Flatow EL, Bigliani LU. The use of arthroscopy in the treatment of resistant frozen shoulder. Clin Orthop Relat Res. 1994;(304):30-36.

99. Klinger HM, Otte S, Baums MH, Haerer T. Early arthroscopic release in refractory shoulder stiffness. Arch Orthop Trauma Surg. 2002; 122(4):200-203.

100. Watson L, Dalziel R, Story I. Frozen shoulder: a 12-month clinical outcome trial. J Shoulder Elbow Surg. 2000;9(1):16-22.

101. Berghs BM, Sole-Molins X, Bunker TD. Arthroscopic release of adhesive capsulitis. J Shoulder Elbow Surg. 2004;13(2):180-185.

102. Nicholson GP. Arthroscopic capsular release for stiff shoulders: effect of etiology on outcomes. Arthroscopy. 2003;19(1):40-49.

103. Ide J, Takagi K. Early and long-term results of arthroscopic treatment for shoulder stiffness. J Shoulder Elbow Surg. 2004;13(2):174-179.

104. Ogilvie-Harris DJ, Biggs DJ, Fitsialos DP, MacKay M. The resistant frozen shoulder. Manipulation versus arthroscopic release. Clin Orthop Relat Res. 1995;(319):238-248.

105. Mehta SS, Singh HP, Pandey R. Comparative outcome of arthroscopic release for frozen shoulder in patients with and without diabetes. Bone Joint J. 2014;96-B(10):1355-1358.

106. Jerosch J, Nasef NM, Peters O, Mansour AM. Mid-term results following arthroscopic capsular release in patients with primary and secondary adhesive shoulder capsulitis. Knee Surg Sports Traumatol Arthrosc. 2013;21(5):1195-1202.

107. Smith CD, Hamer P, Bunker TD. Arthroscopic capsular release for idiopathic frozen shoulder with intra-articular injection and a controlled manipulation. Ann R Coll Surg Engl. 2014;96(1):55-60.

108. Chen J, Chen S, LiY, Hua Y, Li H. Is the extended release of the inferior glenohumeral ligament necessary for frozen shoulder? Arthroscopy. 2010;26(4):529-535.

109. Waszczykowski M, Fabiś J. The results of arthroscopic capsular release in the treatment of frozen shoulder - two-year follow-up. Ortop Traumatol Rehabil. 2010;12(3):216-224. English, Polish.

110. Le Lievre HM, Murrell GA. Long-term outcomes after arthroscopic capsular release for idiopathic adhesive capsulitis. J Bone Joint Surg Am. 2012;94(13):1208-1216.

111. Dattani R, Ramasamy V, Parker R, Patel VR. Improvement in quality of life after arthroscopic capsular release for contracture of the shoulder. Bone Joint J. 2013;95-B(7):942-946.

112. Grant JA, Schroeder N, Miller BS, Carpenter JE. Comparison of manipulation and arthroscopic capsular release for adhesive capsulitis: a systematic review. J Shoulder Elbow Surg. 2013;22(8): 1135-1145.

113. Omari A, Bunker TD. Open surgical release for frozen shoulder: surgical findings and results of the release. J Shoulder Elbow Surg. 2001;10(4):353-357.
Orthopedic Research and Reviews

\section{Publish your work in this journal}

Orthopedic Research and Reviews is an international, peer-reviewed, open access journal focusing on the patho-physiology of the musculoskeletal system, trauma, surgery and other corrective interventions to restore mobility and function. Advances in new technologies, materials, techniques and pharmacological agents are particularly welcome. The journal welcomes

\section{Dovepress}

original research, clinical studies, reviews \& evaluations, expert opinion and commentary, case reports and extended reports. The manuscript management system is completely online and includes a very quick and fair peer-review system, which is all easy to use. Visit http://www.dovepress. com/testimonials.php to read real quotes from published authors. 